Portland State University

PDXScholar

$7-1990$

Reduced Form for Coulomb-wave Multicenter Integrals

Jack C. Straton

Portland State University, straton@pdx.edu

Follow this and additional works at: https://pdxscholar.library.pdx.edu/phy_fac

Part of the Atomic, Molecular and Optical Physics Commons

Let us know how access to this document benefits you.

Citation Details

Straton, Jack C. "Reduced form for Coulomb-wave multicenter integrals." Physical Review A 42 (1990): 307-310.

This Article is brought to you for free and open access. It has been accepted for inclusion in Physics Faculty Publications and Presentations by an authorized administrator of PDXScholar. Please contact us if we can make this document more accessible: pdxscholar@pdx.edu. 


\title{
Reduced form for Coulomb-wave multicenter integrals
}

\author{
Jack C. Straton \\ Department of Physics, Cardwell Hall, Kansas State University, Manhattan, Kansas 66506
}

(Received 28 February 1990)

\begin{abstract}
In a previous paper [J. C. Straton, Phys. Rev. A 41, 71 (1990)] an integro-differential transform was introduced and utilized to obtain the analy tically reduced form for multicenter integrals composed of general-state hydrogenic orbitals, Yukawa or Coulomb potentials, and plane waves. The present paper extends this result to include Coulomb waves.
\end{abstract}

\section{INTRODUCTION}

Theories within atomic and molecular physics generally lead to multicenter integrals containing products of hydrogenic orbitals, Yukawa or Coulomb potentials, plane waves, and Coulomb waves. In a series of papers ${ }^{1-5}$ the author has developed the analytically reduced form for the general case that excludes Coulomb waves. The present paper removes this exclusion. Equations from Refs. 4 and 5 will be referred to by suffixing "I" and "-II," respectively, to the equation numbers.

\section{THE INCLUSION OF COULOMB WAVES}

The general multicenter integral to be reduced is

$$
\begin{aligned}
& S_{I_{1} J_{1} \ldots I_{M} J_{M}}^{\eta_{1} j_{1} \ldots \eta_{M} j_{M}}\left(\mathbf{p}_{1}, \ldots, \mathbf{p}_{m} ; \mathbf{y}_{1}, \ldots, \mathbf{y}_{M} ; \nu_{K}, \mathbf{Q}_{K}, \ldots, \nu_{M}, \mathbf{Q}_{M}\right) \\
& =\int d^{3} x_{1} \cdots d^{3} x_{m} e^{-i\left(\mathbf{p}_{1} \cdot \mathbf{x}_{1}+\cdots+\mathbf{p}_{m} \cdot \mathbf{x}_{m}\right)} P_{I_{1} J_{1}}^{\eta_{1} j_{1}}\left(\mathbf{R}_{1}\right) \cdots P_{I_{K-1} J_{K-1}}^{\eta_{K-1} j_{K-1}}\left(\mathbf{R}_{K-1}\right) \bar{P}_{I_{K} J_{K}}^{\eta_{J_{K}} j_{K}}\left(\mathbf{R}_{K}, \nu_{K}, \mathbf{Q}_{K}\right) \\
& \quad \times \cdots \bar{P}_{I_{M} J_{M}}^{\eta_{M} j_{M}}\left(\mathbf{R}_{M}, \nu_{M}, \mathbf{q}_{M}\right)
\end{aligned}
$$

where

$$
P_{I J}^{\eta j}(\mathbf{R})=u_{I}(\mathbf{R}) \ldots u_{J}(\mathbf{R}) V^{\eta j}(\mathbf{R})
$$

and

$$
\bar{P}_{I J}^{\eta j}(\mathbf{R}, \nu, \mathbf{Q})=P_{I J}^{\eta j}(\mathbf{R})_{1} F_{1}(i \nu, 1, i(Q R+\mathbf{Q} \cdot \mathbf{R}))
$$

in which the $u$ 's are hydrogenic orbitals [(14-II)-(16-II)],

$$
V^{\eta j}(\mathbf{R})=R^{j-1} e^{-\eta R},
$$

and

$$
\mathbf{R}_{i}=\sum_{j=1}^{m} t_{i j} \mathbf{x}_{j}+\sum_{j=1}^{M} u_{i j} \mathbf{y}_{j}
$$

For each confluent hypergeometric function, introduce the real-contoured integral transform ${ }^{6}$

$$
{ }_{1} F_{1}(a, b, z)=\frac{1}{B(a, b-a)} \int_{0}^{1} e^{z \tau} \tau^{a-1}(1-\tau)^{b-a-1} d \tau,
$$

which is more amenable to numerical integration than the more commonly used ${ }^{7-9}$ complex-contoured transform (containing an identical integrand). It will be shown in Sec. III that the former choice gives the same result as the latter.

The final reduced form of the Coulomb-wave integral is

$$
\begin{aligned}
& S_{I_{1} J_{1} I_{M} J_{M}}^{\eta_{1} j_{1} \ldots n_{M} j_{m}}\left(\mathbf{p}_{1}, \ldots, \mathbf{p}_{m} ; \mathbf{y}_{1}, \ldots, \mathbf{y}_{M} ; \nu_{K}, \mathbf{Q}_{K}, \ldots, \nu_{M}, \mathbf{Q}_{M}\right) \\
& =\pi^{3 m / 2} \int_{0}^{\infty} d \rho_{1} \cdots d \rho_{M} \int_{0}^{1} d \tau_{K} \tau^{i \nu_{K}-1}(1-\tau)^{-i \nu_{K}} \\
& \times \cdots \int_{0}^{1} d \tau_{M} \tau^{i \nu_{M}-1}(1-\tau)^{-i \nu_{M}} A_{I_{1} J_{1}}^{n_{1} j_{1}}\left(\rho_{1}, \mathbf{q}_{1}\right) \cdots A_{I_{K-1} J_{K-1}}^{n_{K-1} j_{K-1}}\left(\rho_{K-1}, \mathbf{q}_{K-1}\right) \\
& \times \frac{A_{I_{K} J_{K}}^{n_{J^{\prime}} j_{K}}\left(\rho_{K}, \mathbf{q}_{K}\right) \prod_{j^{\prime}=1}^{M} e^{i u_{K^{\prime}} \tau^{\prime} \tau_{K^{\prime}} \mathbf{Q}_{K} \mathbf{y}_{\jmath^{\prime}}}}{B\left(i \nu_{K}, 1-i \nu_{K}\right)} \\
& \times \cdots \frac{A_{I_{M} J_{M}}^{n_{M} j_{M}}\left(\rho_{M}, \mathbf{q}_{M}\right) \prod_{j^{\prime}=1}^{M} e^{i u_{M j^{\prime}} \tau_{M} \mathbf{Q}_{M} \cdot \mathbf{y}_{\jmath^{\prime}}}}{B\left(i \nu_{M}, 1-i \nu_{M}\right)} \frac{e^{-\Omega / \Lambda}}{\Lambda^{3 / 2}} .
\end{aligned}
$$


The $A$ 's are given by [(24-II)-(29-II)],

$$
\begin{aligned}
& A_{I J}^{\eta j}[\rho, i \mathbf{q}]=\frac{e^{-\gamma^{2} / 4 \rho}}{2^{j} \sqrt{\pi}}\left(\sum_{s_{I}=0}^{n_{I}-\ell_{I}-1} \frac{(-1)^{S_{I}}\left(\lambda_{I} / n_{I}\right)^{s_{I}+\ell_{I}} \lambda_{I}^{3 / 2} N_{n_{I} \ell_{I}}}{\left(n_{I}-\ell_{I}-1-s_{I}\right) !\left(2 \ell_{I}+1+s_{I}\right) ! s_{I} !}\right. \\
& \left.\times \cdots \times \sum_{s_{J}=0}^{n_{J}-\ell_{J}-1} \frac{(-1)^{s_{J}}\left(\lambda_{J} / n_{J}\right)^{s_{J}+\ell_{J}} \lambda_{J}^{3 / 2} N_{n_{J} \ell_{J}}}{\left(n_{J}-\ell_{J}-1-s_{J}\right) !\left(2 \ell_{J}+1+s_{J}\right) ! s_{J} !}\right) \\
& \times \sum_{L_{1}=L_{1} \min }^{L_{1} \max }(-1)^{M_{I+1}}\left(\frac{\left(2 \ell_{I}+1\right)\left(2 \ell_{I+1}+1\right)\left(2 L_{1}+1\right)}{4 \pi}\right)^{1 / 2}\left(\begin{array}{ccc}
\ell_{I} & \ell_{I+1} & L_{1} \\
0 & 0 & 0
\end{array}\right) \\
& \times\left(\begin{array}{rrc}
\ell_{I} & \ell_{I+1} & L_{1} \\
m_{I} & m_{I+1} & -M_{I+1}
\end{array}\right) \\
& \times \sum_{L_{2}=L_{2} \min }^{L_{2} \max }(-1)^{M_{I+2}}\left(\frac{\left(2 L_{1}+1\right)\left(2 \ell_{I+2}+1\right)\left(2 L_{2}+1\right)}{4 \pi}\right)^{1 / 2} \\
& \times\left(\begin{array}{rcc}
L_{1} & \ell_{I+2} & L_{2} \\
0 & 0 & 0
\end{array}\right)\left(\begin{array}{ccc}
L_{1} & \ell_{I+2} & L_{2} \\
M_{I} & m_{I+2} & -M_{I+2}
\end{array}\right) \\
& \times \cdots \times \sum_{L_{J}=L_{J} \min }^{L_{J} \max }(-1)^{M_{J}}\left(\frac{\left(2 L_{J-1}+1\right)\left(2 \ell_{J}+1\right)\left(2 L_{J}+1\right)}{4 \pi}\right)^{1 / 2} \\
& \times\left(\begin{array}{ccc}
L_{J-1} & \ell_{J} & L_{J} \\
0 & 0 & 0
\end{array}\right)\left(\begin{array}{ccc}
L_{J-1} & \ell_{J} & L_{J} \\
M_{J-1} & m_{J} & -M_{J}
\end{array}\right) \\
& \times\left(\frac{2^{L_{J}} H_{s_{I}+\ell_{I}++s_{J}+\ell_{J}-L_{J}+j}(\gamma / 2 \sqrt{\rho})}{\rho^{\left(s_{I}+\ell_{I}+\cdots+s_{J}+\ell_{J}-L_{J}+1+j\right) / 2}} \mathcal{Y}_{L_{J} M_{J}}(i \mathbf{q})\right),
\end{aligned}
$$

where

$$
\begin{aligned}
& M_{j}=m_{I}+m_{I+1}+\cdots+m_{j}, \\
& L_{j \max }=L_{j-1}+\ell_{j}, \\
& \mu_{j}=\max \left(\left|L_{j-1}-\ell_{j}\right|,\left|M_{j-1}+m_{j}\right|\right),
\end{aligned}
$$

and

$$
L_{j \min }=\left\{\begin{array}{l}
\mu_{j} \text { if } L_{j \max }+\mu_{j} \text { is even } \\
\mu_{j}+1 \text { if } L_{j \max }+\mu_{j} \text { is odd }
\end{array}\right.
$$

and where the index "(2)" on the summation sign indicates that one is to sum in steps of 2 .

To account for the Coulomb waves (30-II) must be modified to read

$$
\begin{aligned}
\gamma_{j}= & \lambda_{I_{j}} / n_{I_{j}}+\cdots+\lambda_{J_{j}} / n_{J_{j}}+\eta_{j} \\
& +\left\{\begin{array}{l}
0, \quad j<K \\
-i Q_{j} \tau_{j}, \quad j \geq K .
\end{array}\right.
\end{aligned}
$$

As in (36-II) and (34-II)

$$
\Lambda=\left|\begin{array}{cccc}
a_{11} & a_{12} & \cdots & a_{1 m} \\
a_{21} & a_{22} & & a_{2 m} \\
\vdots & & \ddots & \vdots \\
a_{m 1} & a_{m 2} & \cdots & a_{m m}
\end{array}\right|
$$

where

$$
a_{i j}=\sum_{k=1}^{M} \rho_{k} t_{k i} t_{k j}
$$

From (38-II), (35-II), and (42-II)

$$
\Omega=C \Lambda+\sum_{i=j}^{m} \sum_{j=1}^{m} \mathbf{b}_{i} \cdot \mathbf{b}_{j}(-1)^{i+j+1} \Lambda_{i j}
$$

where $\Lambda_{i j}$ is $\Lambda$ with the $i$ th row and $j$ th column deleted,

$$
C=\sum_{k=1}^{M} \sum_{j=1}^{M} \sum_{j^{\prime}=1}^{M} \rho_{k} u_{k j} u_{k j^{\prime}} \mathbf{y}_{j} \cdot \mathbf{y}_{j^{\prime}}
$$

and

$$
\mathbf{q}_{i}=\frac{1}{\Lambda} \sum_{i^{\prime}=1}^{m} \sum_{j^{\prime}=1}^{m}(-1)^{i^{\prime}+j^{\prime}+1} \Lambda_{i^{\prime} j^{\prime}} t_{i i^{\prime}} \mathbf{b}_{i^{\prime}}
$$

in which the only modification for Coulomb waves is (33II)

$$
\mathbf{b}_{i^{\prime}}=\frac{i \mathbf{p}_{i^{\prime}}}{2}+\sum_{k=1}^{M} \rho_{k} t_{k i^{\prime}} \sum_{j=1}^{M} u_{k j} \mathbf{y}_{j}-\frac{i}{2} \sum_{j=K}^{M} t_{j i^{\prime}} \mathbf{Q}_{j} \tau_{j}
$$


Finally, if the $K$ th $P$ in (1) contains two ${ }_{1} F_{1}$ functions, depending on parameters $\nu, \mathbf{Q}$ and $\nu^{\prime}, \mathbf{Q}^{\prime}$, one must insert

$$
\int_{0}^{1} d \tau_{K}^{\prime} \frac{\tau^{i \nu_{K}^{\prime}-1}\left(1-\tau^{\prime}\right)^{-i \nu_{K}^{\prime}}}{B\left(i \nu_{K}^{\prime}, 1-i \nu_{K}^{\prime}\right)}
$$

in (7), set $\tau_{K} \mathbf{Q}_{K} \rightarrow \tau_{K} \mathbf{Q}_{K}+\tau_{K}^{\prime} \mathbf{Q}_{K}^{\prime}$ in the exponential of (7), and in (19), and set $Q_{K} \tau_{K} \rightarrow Q_{K} \tau_{K}+Q_{K}^{\prime} \tau_{K}^{\prime}$ in (13).

\section{EXAMPLES}

Consider the integral

$$
S^{\eta j}\left(\mathbf{p} ; 0 ; \nu, \mathbf{Q}, \nu^{\prime}, \mathbf{Q}^{\prime}\right)=\int d^{3} x e^{-i \mathbf{p} \cdot \mathbf{x}} x^{j-1} e^{-\eta x}{ }_{1} F_{1}[i \nu, 1, i(Q x+\mathbf{Q} \cdot \mathbf{x})]_{1} F_{1}\left(i \nu^{\prime}, 1, i\left(Q^{\prime}+\mathbf{Q}^{\prime} \cdot \mathbf{x}\right)\right)
$$

In this spherically symmetric case

$$
A^{\gamma j}(\rho, \mathbf{q})=\frac{e^{-\gamma^{2} / 4 \rho}}{2^{j} \sqrt{\pi}} \frac{H_{j}(\gamma / 2 \sqrt{\rho})}{\rho^{(1+j) / 2}}
$$

where

$$
\gamma=\eta-i \tau Q-i \tau^{\prime} Q^{\prime}
$$

Also

$$
\begin{aligned}
& \Lambda=\rho, \quad \Lambda_{11} \equiv 1, \\
& C=0,
\end{aligned}
$$

and

$$
\mathbf{b}=\frac{i}{2}\left(\mathbf{p}-\mathbf{Q} \tau-\mathbf{Q}^{\prime} \tau^{\prime}\right)
$$

so that

$$
\begin{aligned}
\Omega=\frac{1}{4} & \left(p^{2}-2 \tau \mathbf{p} \cdot \mathbf{Q}-2 \tau^{\prime} \mathbf{p} \cdot \mathbf{Q}^{\prime}+Q^{2} \tau^{2}+2 \tau \tau^{\prime} \mathbf{Q} \cdot \mathbf{Q}^{\prime}\right. \\
& \left.+\mathbf{Q}^{\prime 2} \tau^{\prime 2}\right) .
\end{aligned}
$$

For $j=0$ the $\rho$ integral gives

$$
\begin{aligned}
S^{\eta 0}\left(\mathbf{p} ; 0 ; \nu, \mathbf{Q}, \nu^{\prime}, \mathbf{Q}^{\prime}\right) \\
=\frac{4 \pi}{B(i \nu, 1-i \nu) B\left(i \nu^{\prime}, 1-i \nu^{\prime}\right)} \\
\quad \times \int_{0}^{1} d \tau \tau^{i \nu-1}(1-\tau)^{-i \nu} \\
\quad \times \int_{0}^{1} d \tau^{\prime} \frac{\tau^{i \nu^{\prime}-1}\left(1-\tau^{\prime}\right)^{-i \nu^{\prime}}}{\left(\gamma^{2}+\Omega\right)} .
\end{aligned}
$$

The denominator is

$$
\gamma^{2}+\Omega=D+F \tau
$$

where

$$
D=\eta^{2}+p^{2}+2\left(-\mathbf{Q}^{\prime} \cdot \mathbf{p}-i \eta Q^{\prime}\right) \tau^{\prime} \equiv 2 \alpha+2 \beta \tau^{\prime}
$$

and

$$
\begin{aligned}
F & =-2(\mathbf{Q} \cdot \mathbf{p}+i \eta Q)-2\left(Q Q^{\prime}-\mathbf{Q} \cdot \mathbf{Q}^{\prime}\right) \tau^{\prime} \\
& \equiv-2 g-2 d \tau^{\prime}
\end{aligned}
$$

Then the $\tau$ integral gives ${ }^{10}$

$$
\begin{aligned}
& S^{\eta 0}\left(\mathbf{p} ; 0 ; \nu, \mathbf{Q}, \nu^{\prime}, \mathbf{Q}^{\prime}\right) \\
& \quad=\frac{4 \pi}{B\left(i \nu, 1-i \nu^{\prime}\right)} \int_{0}^{1} d \tau^{\prime} \frac{\tau^{i \nu^{\prime}-1}\left(1-\tau^{\prime}\right)^{-i \nu^{\prime}}}{(F+D)^{i \nu} D^{1-i \nu}} .
\end{aligned}
$$

Note that if $\nu=0$, the $\tau^{\prime}$ integral gives

$$
S^{\eta 0}\left(\mathbf{p} ; 0 ; 0, \mathbf{Q}, \nu^{\prime}, \mathbf{Q}^{\prime}\right)=\frac{4 \pi}{(2 \beta+2 \alpha)^{i \nu^{\prime}}(2 \alpha)^{1-i \nu^{\prime}}}
$$

which is the known result ${ }^{11}$ since ${ }_{1} F_{1}(0, b, z) \equiv 1$.

For the general case define

$$
\gamma=g-\alpha, \delta=d-\beta .
$$

Then

$$
D=2 \alpha\left(1+\frac{\beta}{\alpha} \tau^{\prime}\right) \equiv 2 \alpha\left(1-b \tau^{\prime}\right)
$$

and

$$
F+D=-2 \gamma\left(1+\frac{\delta}{\gamma} \tau^{\prime}\right) \equiv-2 \gamma\left(1-a \tau^{\prime}\right) .
$$

Changing variables to

$$
t=\tau^{\prime}\left(\frac{1-a}{1-a \tau^{\prime}}\right)
$$

where

$$
\begin{aligned}
S^{\eta 0}\left(\mathbf{p} ; 0 ; \nu, \mathbf{Q}, \nu^{\prime}, \mathbf{Q}^{\prime}\right)= & \frac{4 \pi(2 \alpha)^{i \nu-1}(-2 \gamma)^{-i \nu}}{B\left(i \nu^{\prime}, 1-i \nu^{\prime}\right)}(1-a)^{1-i \nu^{\prime}-i \nu+i \nu-1} \\
& \times \int_{0}^{1} d t t^{-2+i \nu^{\prime}+1}(1-t)^{-i \nu^{\prime}}(1-z t)^{i \nu-1}(1-a+a t)^{-\imath \nu^{\prime}-1+i \nu^{\prime}+i \nu-i \nu+1},
\end{aligned}
$$

$$
z=\frac{b-a}{1-a}=\frac{\alpha \delta-\beta \gamma}{\alpha(\gamma+\delta)} .
$$


The $t$ integral may be evaluated ${ }^{12}$ giving

$$
S^{\eta 0}\left(\mathbf{p} ; 0 ; \nu, \mathbf{Q}, \nu^{\prime}, \mathbf{Q}^{\prime}\right)=\frac{2 \pi}{\alpha} e^{-\pi \nu}\left(\frac{\alpha}{\gamma}\right)^{i \nu}\left(\frac{\gamma+\delta}{\gamma}\right)^{-i \nu^{\prime}} F\left(1-i \nu, i \nu^{\prime}, 1, z\right)
$$

where the factor $e^{-\pi \nu}$ arises from the identification $(1 /-1)^{i \nu}=\left(-1 /(-1)^{2}\right)^{i \nu}=\left(e^{i \pi} / 1\right)^{i \nu}$. This is Nordsieck's result ${ }^{7}$ if one sets $\mathbf{Q} \rightarrow-\mathbf{p}_{1}, \mathbf{Q}^{\prime} \rightarrow \mathbf{p}_{2}, \mathbf{p} \rightarrow-\mathbf{q}, \nu \rightarrow a_{1}, \nu^{\prime} \rightarrow a_{2}$, and $\eta \rightarrow \lambda$.

Note that the limits of (33) and (40) are well defined for $\eta \rightarrow 0$ when factors of $(-1)^{i \nu}$ are properly accounted for. ${ }^{8}$ Thus the Gausian transform ${ }^{13}$ leading to (8) is well defined in the limit $\operatorname{Re} \gamma \rightarrow 0$ for integrals of this type.

\section{CONCLUSION}

The analytically reduced form has been found for the general multicenter integral of products of hydrogenic orbitals, Yukawa or Coulomb potentials, plane waves, and Coulomb waves. It has been shown that the introduction of a real-contoured integral transform for the confluent hypergeometric function, which is more amenable to numerical evaluation than the more common complex- contoured integral transform, gives the known analytical result for Nordsieck's integrals.

\section{ACKNOWLEDGMENTS}

This work was supported by the Division of Chemical Sciences, Office of Energy Research, U.S. Department of Energy. Helpful discussions with J.H. McGuire are appreciated.
${ }^{1}$ J.C. Straton, Phys. Rev. A 35, 2729 (1987); 37, 4531 (1988).

2 J.C. Straton, Phys. Rev. A 39, 5062 (1989).

${ }^{3}$ J.C. Straton, Phys. Rev. A 39, 5071 (1989).

${ }^{4}$ J.C. Straton, Phys. Rev. A 39, 1676 (1989); 40, 2819 (1989).

${ }^{5}$ J.C. Straton, Phys. Rev. A 41, 71 (1990).

${ }^{6}$ Handbook of Mathematical Functions with Formulas, Graphs, and Mathematical Tables, Natl. Bur. Stand. Appl. Math. Ser. No. 55, edited by M. Abramowitz and I.A. Stegun (U.S. GPO, Washington, D.C., 1972), p. 505, No. 13.2.1.

${ }^{7}$ A. Nordsieck, Phys. Rev. 93, 785 (1954).

${ }^{8}$ M.R.C. McDowell and J.P. Coleman, Introduction to the Theory of Ion-Atom Collisions (North-Holland, Amsterdam, 1970), pp. 364-370.

${ }^{9}$ M. Brauner, J.S. Briggs, and H. Klar, J. Phys. B 22, 2265
(1989).

${ }^{10}$ I.S. Gradshteyn and I.M. Ryzhik, Table of Integrals, Series, and Products (Academic, New York, 1980), p. 287, No. 3.198; or W. Grobner and N. Hofreiter, Integraltafel Zweiter Teil Bestimmte Interale (Springer-Verlag, Berlin, 1961), p. 175, No. 421.4.

${ }^{11}$ J.P. Coleman, in Case Studies in Atomic Collision Physics, edited by M.R.C. McDowell and E.W. McDaniel (NorthHolland, Amsterdam, 1969), Vol. 1, pp. 161-163.

${ }^{12}$ I.S. Gradshteyn and I.M. Ryzhik, Table of Integrals, Series, and Products (Academic, New York, 1980), p. 1040, No. 9.111 .

${ }^{13}$ I.S. Gradshteyn and I.M. Ryzhik, Table of Integrals, Series, and Products (Academic, New York, 1980), p. 340, No. 3, 471(9); or derivatives with respect to $b$ of W. Grobner and N. Hofreiter, Integraltafel Zweiter Teil Bestimmte Integrale (Springer-Verlag, Berlin, 1961), p. 66, No. 9c. 